\title{
Need for Consensus Statement for Cholangiocarcinoma Diagnosis, Prognostication and Treatment: An IASGO Initiative
}

\author{
Nuh N. Rahbari ${ }^{1}$, Dan G. Duda ${ }^{2}$, Mitesh J. Borad ${ }^{3}$, Ho-Seong Han ${ }^{4}$
}

'Department of Surgery, Universitätsmedizin Mannheim, Medical Faculty Mannheim, Heidelberg University, Mannheim, Germany

'Edwin L. Steele Laboratories for Tumor Biology, Department of Radiation Oncology, Massachusetts General Hospital, Boston, USA

${ }^{3}$ Divison of Hematology/Oncology, Mayo Clinic, Phoenix, USA

${ }^{4}$ Seoul National University Bundang Hospital, Seongnam, Seoul, South Korea

While relatively infrequent, cholangiocarcinomas (CCAs) are liver cancers whose incidence is increasing worldwide. Unfortunately, the progress in medical, surgical and radiation oncology treatment of CCA has made little strides in the survival of patients in most regions of the world over the past several decades. As a result, CCA therapy remains a major challenge in medicine. There are multiple reasons for limited advances in CCA care. These include: (1) the lack of standardized surgical approaches, (2) the relative difficulty of conducting clinical trials due to the low incidence of this malignancy, (3) the typically late diagnosis of CCA, a disease with aggressive local growth and early metastatic spread, (4) resistance to cytotoxic chemotherapy and to many molecularly targeted agents, (5) difficulties in implementing radiation therapy protocols, (6) heterogeneity of oncogenic drivers and differences between CCAs based on their localization and (7) lack of biomarkers to guide therapy. Nevertheless, there have been recent advances in our understanding of the disease coming from genomic studies, in implementation of new and improved surgical and radiotherapy protocols, and promising data from early clinical trials of targeted agents and immunotherapy.

In an attempt to highlight this progress, to better understand the reasons for the differences in patient care across continents, and to define the key questions for future clinical trials in CCA, IASGO initiated a consensus meeting that took place at Seoul National University Bundang Hospital in Seoul, Korea on June 29 and 30, 2018. The meeting included a large number of experts in CCA, specialized in tumor biology, pathology, surgery, radiology, radiation oncology and medical oncology. At the conclusion of this meeting, the participants agreed that a broad and globally applicable consensus may be difficult to achieve on all areas of CCA care. The group also decided to put together a 'white paper' that outlines different approaches of CCA management with the aim to make specific recommendations and initiate prospective trials addressing controversial topics. This white paper will be published in a future issue of Surgery, Gastroenterology and Oncology.
Corresponding author:

Nuh N. Rahbari, MD

E-mail: nuh.rahbari@umm.de
Copyright (1) Celsius Publishing House www.sgo-iasgo.com 
In this special issue of Surgery, Gastroenterology and Oncology, we focused on these advances in the contemporary management of CCA and discuss future directions in this important area of unmet need. We are grateful to the experts on CCA management from leading institutions in the US, Europe, North Africa and Asia who reviewed current concepts of diagnosis and treatment in an attempt to provide benchmarks and recommendations for the optimal therapy of patients suffering from CCA. These reports clearly demonstrate the complexity of CCA management, and the importance of multidisciplinary approaches. They also emphasize once more the significant diversity of thera- peutic approaches between Eastern and Western institutions, a hallmark of CCA treatment.

This IASGO initiative is certainly only the beginning of a long process and complex conversation between experts of multiple disciplines from all over the world. We think that there is no better forum for this difficult task than IASGO, given its global reach and mission, and its multidisciplinary approach to improved healthcare for all. We sincerely hope that this effort will translate very soon into better education and more information for the specialists treating this dreadful disease and ultimately, in better and longer life for CCA patients worldwide. 